\title{
Event report: esss 2011-Scientometric education in Indian summer at the University of Vienna
}

\author{
Christian Gumpenberger • Juan Gorraiz • Wolfgang Glänzel • \\ Koenrad Debackere $\cdot$ Stefan Hornbostel $\cdot$ Sybille Hinze
}

Received: 30 November 2011/Published online: 21 December 2011

(C) Akadémiai Kiadó, Budapest, Hungary 2011

According to the annual rotation idea the second esss (European Summer School for Scientometrics) was hosted by the University of Vienna from 11 to 16 September 2011. It was again jointly organized by the University of Vienna (Austria), the Humboldt University (Germany), the Katholieke Universiteit Leuven (Belgium) and the iFQ (Institute for Research Information and Quality Assurance, Germany).

While last year's debut programme in Berlin (Gorraiz et al. 2010a, b) comprised of only 3 days, this year's event was expanded to 6 days including a free pre-programme, a conference, seminars, hands-on sessions and a workshop. The pre-programme as well as the conference took place at the appealing reading room of Teinfaltstrasse Library, which is part of the Vienna University Library. esss seminars, hands-on sessions and the workshop were then all held at the IT Seminar Rooms of the Vienna University Campus.

esss 2011 started unofficially with a free pre-programme on 11 September, which was a bibliometric crash course for esss participants short on experience in the field. The attendees were familiarized with the main terms and concepts of bibliometrics by a panel of experts composed of Wolfgang Glänzel (KU Leuven, Belgium), Sybille Hinze (iFQ, Germany), Juan Gorraiz and Christian Gumpenberger (both University of Vienna, Austria).

The official opening of esss 2011 took place on 12 September by the newly appointed Vice Rector Susanne Weigelin-Schwiedrzik from the University of Vienna, who emphasized the importance of bibliometrics and gave a warm welcome to the 120 attendees of the 2 days esss conference.

C. Gumpenberger · J. Gorraiz

University of Vienna, Vienna, Austria

W. Glänzel $(\bowtie) \cdot$ K. Debackere

Katholieke Universiteit Leuven, Leuven, Belgium

e-mail: Wolfgang.Glanzel@econ.kuleuven.ac.be

S. Hornbostel $\cdot$ S. Hinze

Institute for Research Information and Quality Assurance (iFQ), Berlin, Germany

S. Hornbostel

Humboldt University of Berlin, Berlin, Germany 
Conference day 1 was dedicated to "Theoretical and Practical Aspects" of Scientometrics. Wolfgang Glänzel and Stefan Hornbostel (iFQ, HU Berlin, Germany) as the first speakers provided a concise overview of the "History and Institutionalization of Scientometrics", followed by Anthony van Raan (Leiden University, the Netherlands) whose talk "New developments in bibliometric methods for evaluation and mapping of scientific research" included opinions on bibliometric "mortal sins" that were entertaining and controversial at the same time. Werner Marx's (Max Planck Institute for Solid State Research, Stuttgart, Germany) talk about "Bibliometrics in the History and Philosophy of Science" then clearly demonstrated that bibliometrics is certainly not strictly limited to evaluation purposes. However, Henk Moed (Elsevier, the Netherlands) brought the audience back to reality and presented "New developments in the use of bibliometric tools in research assessment".

In the afternoon Koenraad Debackere (KU Leuven, Belgium) talked about "Practical Aspects of Scientometrics" and reflected on imperatives and indicators of modern science and innovation policy. Jonathan Adams (Research Evaluation, Thomson Reuters, UK) picked up where his previous speaker left off and presented "Bibliometrics, Research Evaluation and National Science Policy: What works and what goes wrong?". The final talks of the first day were given by representatives of Elsevier B.V. (Netherlands). Arthur Eger first provided an easy-to-follow "Scopus in-person tutorial", continued by Jörg Hellwig who brought along a fancy presentation of "The SciVal Suite-Helping institutions to establish, evaluate and execute research strategies".

The overall topic of conference day 2 (13 September) was "Procedures and Indicators". Wolfgang Glänzel and Sybille Hinze set the stage and introduced "Metrics for Research Evaluation: Indicators, Methods and Mathematical Foundations". The next two presentations were dedicated to the visualization trend in the discipline. Bart Thijs (KU Leuven, Belgium) and Edgar Schiebel (AIT, Austria) talked about background and techniques of "Mapping Science" and "Network Analyses".

After the lunch break Matthias Winterhager (Bielefeld University, Germany) showed quite plainly "What to do (and not to do) with corporate data" and pointed out the wellknown issues with affiliations. And since one of the major requirements for bibliometric analyses are appropriate "Bibliometric Data Sources", Wolfgang Glänzel introduced the "usual suspects" (Web of Science, Scopus, Google Scholar), whereas Juan Gorraiz focused on less obvious alternative citation sources like MathSciNet, CAS SciFinder, Derwent Innovations Index, Espacenet, ArXiv.org, Citebase and CiteSeerX.

Like the day before the remaining afternoon was reserved for presentations from industry. Jeff Clovis' (Thomson Reuters, Philadelphia) first talk "Creating and Supporting Innovative Research Pathways" was intended to familiarize the esss attendees with the new interface of Web of Science, whereas the second talk "Assessing faculty productivity and institutional research performance: Using publication and citation key performance indicators" gave an overview of the portfolio of Thomson Reuters products geared towards research assessment.

The last 3 days (14-16 September) were all about what a summer school is meant to be: seminars, hands-on sessions, group work and presentations. Due to the high demand the organizers doubled the number of accepted participants from 25 to 50 this year, who were split into two groups taught in parallel sessions.

14 September was dedicated to "Bibliometric Indicators". Wolfgang Glänzel and Juan Gorraiz talked about "Journal Impact Measures", whereas Jeffrey Demaine (iFQ, Germany) and William Dinkel (GESIS, Germany) presented "h-index and related measures" 
in the morning. The imparted theory was then consolidated in practical hands-on exercises in the afternoon.

The focus topics of 15 September were "Cooperation, Co-authorship, Social Networks" on the one hand, and "Mapping Science" on the other. The first topic was taught by András Schubert (Hungarian Academy of Sciences, Hungary), who managed to also add a few anecdotes to his lecture. The second topic was presented by this year's De Solla Price Medal winner Olle Persson, whose lecture based on his software Bibexcel was exciting but challenging to follow due to Persson's speed and enthusiasm.

As a special treat a guided tour through the premises of the Austrian National Library concluded this day, where the esss participants could marvel at the beauty of the impressive State Hall and glimpse at the biggest collection of globes worldwide located at the Globe Museum.

16 September was the final day of the esss course and designed as a workshop titled "Research Evaluation in Practice". After a shared introduction for all by Wolfgang Glänzel, the participants were asked to work on specific tasks in small groups. Two topics were given (1. Bibliometrics-Citation Analysis, 2. Neurobionics), and the participants had to determine the most important players, the most frequent journals and subject categories in the field and the topics within the field and their distribution. Furthermore they should determine basic indicators of citation impact and analyse the h-core. Then an international collaboration analysis should be performed and the 5 most productive countries as well as the main partners identified. Finally publication activity and citation impact of the five most productive countries in the field should be determined.

Group work was individually supported by Wolfgang Glänzel, Sybille Hinze, Juan Gorraiz, Stefan Hornbostel, Jeffrey Demaine and Ambros Wernisch (University of Vienna, Austria).

After a hearty lunch break at the Universitätsbräu on the Campus in beaming sunshine, all participants regathered in the big forum to present their results. The organizers were happy to see the high quality of the presentations which proved that the participants actually used what they had learned during the whole week.

Overall esss 2011 was a huge success with highly positive and encouraging feedback from the participants, who came to Vienna from 22 countries (Algeria, Australia, Austria, Belgium, Colombia, Czech Republic, Denmark, Estonia, France, Germany, Italy, Lebanon, Lithuania, Netherlands, Norway, Poland, Saudi Arabia, South Korea, Sweden, Thailand, United Kingdom, United States). Thus the European Summer School for Scientometrics has already become a truly international event in the second round, and the organizers are looking forward to welcome the next participants for esss 2012 held at the Katholieke Universiteit Leuven in Belgium in the first week of July. Further announcements will be made via esss website (www.scientometrics-school.eu) and via the esss mailing list (to register please send an informal email to office@scientometrics-school.eu).

\section{References}

Gorraiz, J., Gumpenberger, C., Glänzel, W., Debackere, K., Hornbostel, S., \& Hinze, S. (2010a). esss 2010: A review of the inaugurational European Summer School for Scientometrics in Berlin. Scientometrics, 86(1), 235-236. doi:10.1007/s11192-010-0279-4.

Gorraiz, J., Gumpenberger, C., Hornbostel, S., Hinze, S., Glänzel, W., \& Debackere, K. (2010b). European Summer School for Scientometrics (esss) to be launched. Scientometrics, 83(2), 601-602. doi: 10.1007/s11192-010-0206-8. 